\title{
Progress status in fabrication of HBC stripper foil for 3-GeV RCS at J-PARC in Tokai site
}

\author{
Masahiro Yoshimoto*, Yoshio Yamazaki, Takamitsu Nakanoya, Pranab Kumar Saha, and Michikazu Kinsho \\ JAEA/J-PARC, Japan Atomic Energy Agency, 2-4 Shirane, Shirakata, Tokai, Naka-gun, Ibaraki, 319-1195, Japan
}

\begin{abstract}
In the 3-GeV Rapid Cycling Synchrotron (RCS) at Japan Proton Accelerator Research Complex (J-PARC), we adopted thick Hybrid type Boron-doped Carbon (HBC) stripper foil for multi-turn $\mathrm{H}^{-}$charge-exchange injection. The HBC stripper foil developed at KEK has demonstrably improved the foil lifetime significantly. In addition, Graphite Thin Films (GTF) developed by KANEKA corp. was attempted to use for user operation. The GTF is a multilayer graphene sheet and it is expected to be a new candidate for highly durable charge stripping foil. HBC and GTF foils can be both employed the J-PARC RCS for long-term user operation. Early manufacturing of the stripper foil for the J-PARC was carried out according to the following two steps: foil fabrication at the KEK Tsukuba site and foil preparation at the JAEA Tokai site. Recently, the HBC foil deposition apparatus was relocated from KEK to JAEA, and new HBC foils were fabricated at the Tokai site. The performance of the new HBC foil was verified to be equivalent to the original $\mathrm{HBC}$ foil with offline beam irradiation tests. Furthermore, the use of the new HBC foils was successfully demonstrated during RCS user beam operation for one week.
\end{abstract}

\section{Introduction}

The Japan Proton Accelerator Research Complex (J-PARC) is a multipurpose high intensity proton accelerator facility $[1,2]$. It is a joint project between Japan Atomic Energy Agency (JAEA) and High Energy Accelerator Research Organization (KEK), and it is located in Tokai-Mura, Japan. J-PARC consists of three accelerators, namely, a $400-\mathrm{MeV}$ LINAC, a $3-\mathrm{GeV}$ Rapid Cycling Synchrotron (RCS), and a 30-GeV Main Ring Synchrotron (MR), and three experimental facilities, namely, a Materials and Life Science Experimental Facility (MLF), a Hadron Experimental Hall (HD), and a Neutrino Beam Line to the SuperKamiokande (NU). In this chain of accelerators, the RCS performs two functions; as a proton driver to produce pulsed muons and neutrons at MLF and as an injector to $\mathrm{MR}$, aiming at $1 \mathrm{MW}$ output beam power. To achieve MW class high power proton beams in at J-PARC, the multi-turn charge-exchange $\mathrm{H}^{-}$beam injection scheme with stripper foils has been adopted for RCS.

In RCS, a thick Hybrid type Boron-doped Carbon (HBC) stripper foil has been in mainly use since the start of early beam commissioning in 2007 [3]. The HBC stripper foil was developed by Sugai at KEK to drastically improve foil lifetime [4]. The HBC foil technology is patented [5], where the original assignee is KEK, and the inventors are I. Sugai, M. Oyaizu, and H. Takeda. Then, JAEA entered into a licensing arrangement with KEK for the HBC foil in 2018. The $\mathrm{HBC}$ foil deposition apparatus was relocated from the KEK Tsukuba site to the JAEA Tokai site, and we have started fabrication of a new HBC foil. (We call the new foil fabricated at the JAEA Tokai site J-HBC foil.).

In this paper, we report the satisfactory results of the original HBC foil obtained over long-term user operation of RCS. In addition, the results of various offline tests for the performance evaluation of the new J-HBC foil and trial use in J-PARC RCS are reported.

\section{Stripper foil in J-PARC RCS}

The stripper foil plays a very important role in a high power proton beam accelerator, and it is one of the most important components in J-PARC. The HBC stripper foil, which is produced by the arc-discharge method with carbon electrodes doped with boron carbide $\left(\mathrm{B}_{4} \mathrm{C}\right)$ particles, has adequate toughness to withstand highintensity beam irradiation. Indeed, more than 10 sheets of HBC foils have been used in user beam operation, and all of them have withstood beam irradiation thus far. Therefore, we consider that the HBC foil has achieved satisfactory results in the J-PARC. By contrast, Graphite Thin Films (GTF) supplied by KANEKA Corp. [6, 7] have been trialled recently. GTF is a multilayer graphene sheet and it is prepared by annealing a thin polyimide film at temperatures of approximately $2900{ }^{\circ} \mathrm{C}$ in vacuum, and it is characterized by high tensile strength of approximately $40 \mathrm{MPa}$. The preparation process endows GTF with excellent mechanical robustness and flexibility, high purity, and superior heat resistance. Thus, GTF is expected to be a new candidate for highly durable charge stripping foils. Indeed, the GTF stripper foil is installed in the RCS and used for user operation.

*Masahiro Yoshimoto: yoshimoto.masahiro@jaea.go.jp 
As the result, it has been verified to have high robustness against high power beam irradiation, but, at the same time, an issue which is discussed below has been found in the RCS.

The beam commissioning of the RCS for initial beam tuning started in October 2007, and user operation with an output power of $4 \mathrm{~kW}$ started in December 2008 [8]. Since then, the output beam power has been increasing smoothly with beam tuning to reduce beam losses. Two important milestones at J-PARC from the viewpoint of increasing the output beam power are the following [9]: the energy upgrade of LINAC from $181 \mathrm{MeV}$ to $400 \mathrm{MeV}$ during the long shutdown in 2013, and the peak current upgrade from $30 \mathrm{~mA}$ to $50 \mathrm{~mA}$ by renovating the front end devices in the LINAC during the summer shutdown in 2014. After the two important upgrades, continuing efforts to increase the output beam power while minimizing beam losses have been sustained. The first 1-MW beam trial test was performed successfully in October 2014, immediately after the injection beam current upgrade and output beam power for user operation was stood at up to $500 \mathrm{~kW}$ in 2015 . After performing fine beam tuning tests to reduce beam losses, 1-MW beam operation at $25 \mathrm{~Hz}$ is now estimated to be realized sufficiently within a permissible beam loss level [10].
The HBC stripper foils have been supporting the RCS beam tuning and user operation over a period of 10 years. Figure 1 shows a summary of the actual accomplishments of HBC stripper foils in the RCS since recovery from the huge earthquake in 2011 until the summer shutdown in 2016. The upper horizontal bar chart shows the status of user operation. The middle two plots show as follows: the trend of the number of injected $\mathrm{H}^{-}$particles into the RCS every hour, indicating the total output beam power of RCS beam operation; the trend of integral of the injected $\mathrm{H}^{-}$particles irradiating onto each stripper foil. However, the actual magnitude of beam irradiation onto each foil is five to seventy times larger than integral value in the graph because not only the injected $\mathrm{H}^{-}$beam but also the circulating proton beam irradiate onto the foil [10]. The lower photos show the changes in the stripper foils owing to beam irradiation. The stripper foils from ID (a) to ID (h) are the HBC foils, and ID (i) is the GTF foil. The stripper foils have been deformed by beam irradiation, but none of them got broken during beam irradiation thus far. In most cases, irradiated foils have been replaced with new ones during every summer shutdown as a precautionary measure to avoid the spread of broken fragments of highly activated stripper foils in the beam ducts.


Fig. 1. History of beam power increase and stripper foil usage in the J-PARC RCS from 2011 to 2016. 
In J-PARC RCS, the stripper foil is semi selfstanding and is not restricted with the foil frame. Therefore, observed transformation due to beam irradiation shows interesting properties of the foil itself. The shape and the surface of the HBC foils show drastic changes. The irradiation spot is flattened, and the micro bump structure on the surface disappeared. By contrast, the surrounding areas were shrunken and wavy in appearance. It is assumed that the wave appearance can be scribed by pulling from the irradiation spot. However, these drastic changes have not affected the charge exchange property of the foils. On the other hand, the changes in the GTF stripper foil are different from those in the HBC foils. The GTF stripper foil was installed in the RCS in September 2016 and has been employed for user operation with an output beam power of $150 \mathrm{~kW}$ for 4 months. The beam irradiation spot shows almost no changes. The charge exchange efficiency shifted gradually for that period, but the changes does not contribute to stop the user operation. The foil was confirmed to be robust against high power beam irradiation. However, the area surrounding the beam irradiation spot is bent, and especially, the edge of the foil is curved significantly. The GTF foil is a multi-layer graphene sheet, and it has a very ordered structure. Therefore, effect of material damages, such as lattice defects caused by beam irradiation, cannot be contained locally, and they spread across the entire foil in the form of internal stresses. Curving of the foil edge is a critical issue for RCS because it leads to leak the injecting $\mathrm{H}^{-}$ particles without hitting the foil and to increased foil scattering beam losses. Thus, a foil lifetime for charge exchange beam injection should be determined by the foil curving. To evaluate the actual lifetime of the GTF stripper foil, we must continue our investigation of its use in user beam operation.

\section{Fabrication of New HBC foil for J-PARC}

\subsection{Manufacturing process of $\mathrm{HBC}$ foils}

Stable supply of the stripper foils is important from the viewpoint of sustaining stable beam operation in J-PARC. Early manufacturing process of the HBC stripper foil at J-PARC was carried out according to the following two steps, as shown in figure 2: foil fabrication at KEK Tsukuba site and foil preparation at JAEA Tokai site [11].

The foil fabrication step consists of three processes. For pre-processing, a glass plate is coated with a release agent called "Crème-Cote" [12] to release the deposited foil from the glass plate easily. The main processing is carbon evaporation by using the arc-discharge method. By using the boron doped carbon electrodes, HBC foils can be fabricated. After evaporation processing, the foils deposited on the glass is annealed at approximately $470 \mathrm{~K}$ for $2 \mathrm{~h}$. This post-processing is performed to release the high residual mechanical stresses in the deposited foil. In addition, it is an important processing for reduction the curving of the foil due to beam irradiation.

The foil preparation step consists of five processes. For releasing the deposited foil, the glass plate is placed carefully in pure water to float the foil on the water. Therefore, the floating foil is collected from the water. By pulling up the center line of the foil, a double-layer structure can be created [13]. After drying, the collected foil is placed between two pieces of thin tracing paper and is cut into the desired size by using scissors. Finally, a pair of U-shaped aluminium frame is used, with bonded SiC fibers of $7 \mu \mathrm{m}$ diameter, and the foil is mounted on the frame [14]. Fifteen stripper foils on the frames are set in the frame rack and installed in the vacuum chamber as spare foils during user operation.
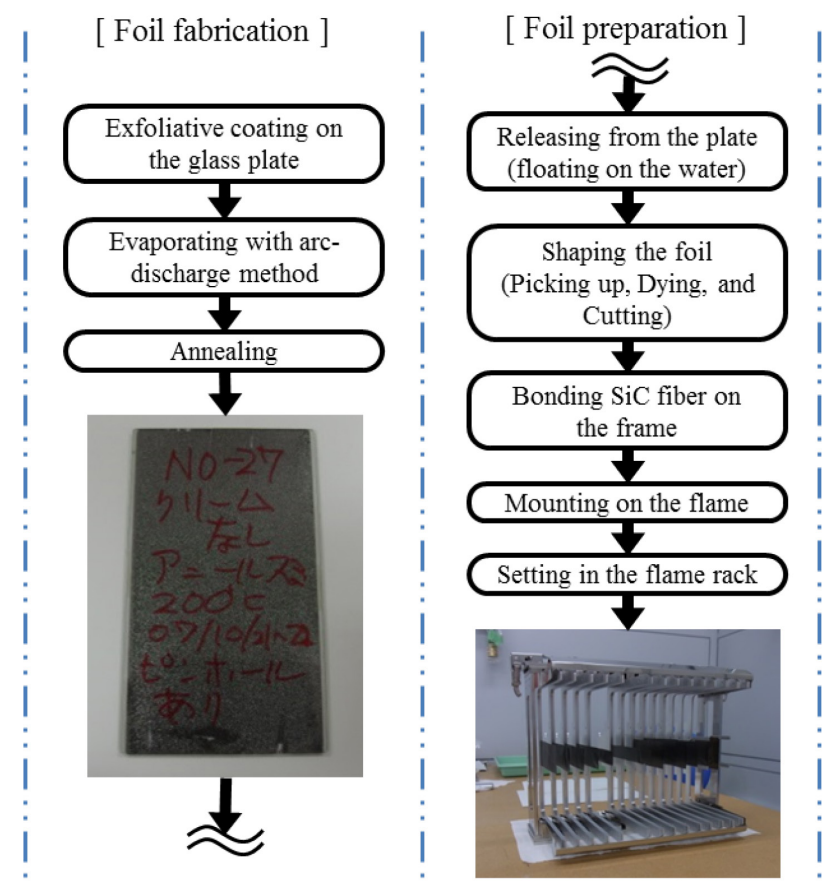

Fig. 2. Schematic diagram of foil manufacturing process.

\subsection{Relocation and restoration of deposition apparatus for HBC foil}

The foil fabrication step significantly influences the performance of the HBC foil. Therefore, it was executed recently at the KEK Tsukuba site by Sugai who is the inventor of and an expert on the HBC foils. However, it was difficult to fabricate the HBC foils at KEK. To maintain a stable supply of the HBC foils at J-PARC, there was a need to quickly restart foil fabrication at JAEA Tokai site. Before starting work on the HBC foil fabrication, JAEA entered into a licensing arrangement with KEK for manufacturing the $\mathrm{HBC}$ foils. Subsequently the device transfer procedure from KEK to JAEA was completed. Then, the deposition apparatus for the HBC foils was relocated from the Tsukuba site to the Tokai site.

This apparatus had been left unused without any maintenance for a considerable period since it was last used. Therefore, the equipment exhibited multiple problems, such as water leak, vacuum leak, metal 
corrosion, and dielectric breakdown. After relocation, machine maintenance, part replacement, and overhaul were performed. Devices with unnecessary functions were removed. As a consequence of many efforts, the deposition apparatus was restored successfully after one year interruption. Figure 3 shows the restored deposition apparatus after it was overhauled.

Some vapor deposition conditions for new foil fabrication are different from the original conditions because of repair and overhaul of the deposition apparatus. Furthermore, detailed procedure to fabricate the HBC foils prescribed by Sugai is not available. In such an environment, we started an attempt to fabricate the new foil at the Tokai site by recalling how he had fabricated the HBC foils previously. And the vacuum components were treated with extra care to prevent any contamination. For example, the glass plates were washed with an ultrasonic cleaning machine, and the deposited foils both before and after their release from the glass plate were stored in a desiccator. After several trial deposition tests, we successfully fabricated a new HBC foil at the JAEA Tokai site. (As mentioned before, we call the new foils J-HBC foil.) Subsequently, the performance of the J-HBC foil was evaluated before installation in the RCS and to use for the user operation.

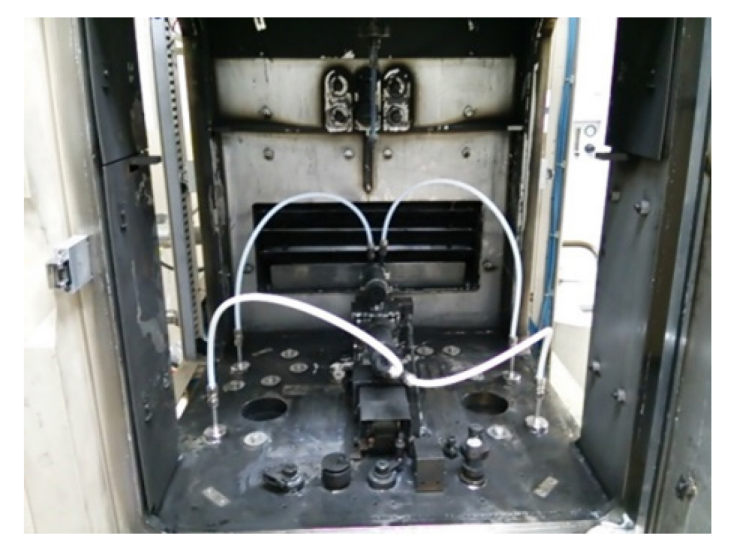

Fig. 3. Photograph of restored deposition apparatus for $\mathrm{HBC}$ foil based on EBX-1000 system.

\subsection{Performance evaluation of J-HBC foil}

The original HBC foil was developed to improve foil lifetime against high power beam irradiation and it can achieve actual accomplishments at the long-term user operation in the J-PARC RCS. By contrast, we still cannot understand completely why the carbon foils can obtain higher durability against beam irradiation by boron-doping. To analyse the $\mathrm{HBC}$ foil by various methods and to understand the mechanism of foil breakage owing to beam irradiation, we have been conducting offline beam tests at Takasaki Ion Accelerators for Advanced Radiation Application (TIARA) in National Institutes for Quantum and Radiological Science and Technology (QST) [11, 14].

Therefore, same offline tests could be conducted for performance evaluation of the J-HBC foil by comparing the test results with those obtained using the original $\mathrm{HBC}$ foils. Hence, the following four offline tests were performed to evaluate the performance of the J-HBC foil: (1) surface microstructural observation by means of Scanning Electron Microscopy (SEM), (2) elemental composition analysis and foil thickness estimation by using the Rutherford Backscattering Spectrometry (RBS) method, (3) identification of trace impurities in the foil by using the micro-Particle Induced X-ray Emission ( $\mu$ PIXE) method, (4) endurance test and foil lifetime estimation by using argon ion beam irradiation and surface observation by digital microscope.

\subsubsection{Surface microstructural observation by SEM}

The HBC foil based on the cluster carbon foil was fabricated using the ACDC arc-discharge method [15]. Therefore, the HBC foil consists of amorphous carbon, and it has a cluster structure with mixed large clusters of $300 \pm 200 \mathrm{~nm}$ derived from the cathode electrode and small clusters of $3 \pm 2 \mathrm{~nm}$ derived from the anode electrode. Figure 4 shows a comparison of the SEM images (JEOL JSM-6700F) of the original-HBC foil and J-HBC. The cluster structure of the J-HBC foil was found to be reasonable compared to that of the originalHBC foil.
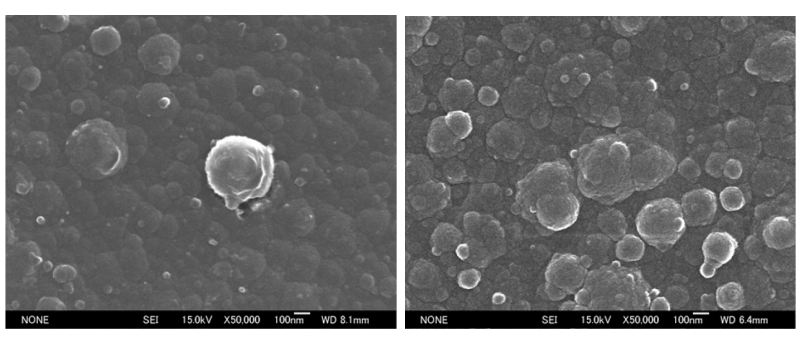

Fig. 4. Comparison of SEM images between original HBC foil and J-HBC foil. (left: original-HBC, right: J-HBC)

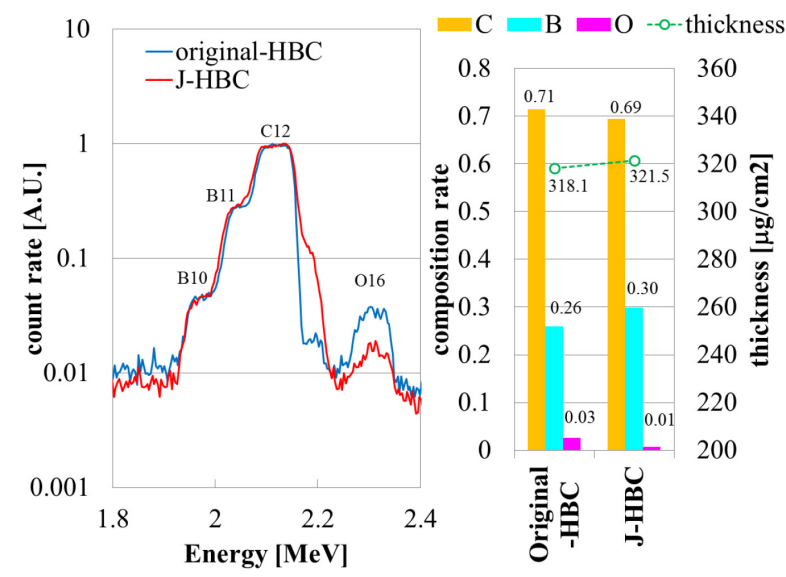

Fig. 5. Comparison of RBS spectra of original HBC foil and J-HBC foil (left), and analysis results for composition ratio and foil thickness estimation (right).

\subsubsection{Elemental composition analysis and thickness estimation by RBS}

The major elemental composition of the HBC foils was analysed using the RBS method with a proton beam of $3 \mathrm{MV}$ in the TIARA 3-MV single-ended accelerator. The 
left graph in figure 5 shows a comparison of the RBS spectra of the original-HBC foil and the J-HBC foil. It is possible to obtain an analysis result for boron-to-carbon ratio and foil thickness estimation as shown the right bar chart of figure 5, by using SIMNRA backscattering spectra simulation code [16]. Both foils achieved the designed foil thickness of $330 \pm 33 \mu \mathrm{g} / \mathrm{cm}^{2}$. However, the boron-to-carbon ratios of the original-HBC and the $\mathrm{J}-\mathrm{HBC}$ foils were slightly different. The cause of this difference is the electrode combination set in the deposition apparatus. In case of the original-HBC foil, the cathode electrode was a boron-doped (25\%) carbon rod of $10 \mathrm{~mm}$ in diameter while the anode electrode was a pure graphite rod of $15 \mathrm{~mm}$ in diameter. However, in case of the J-HBC foil, both electrodes were borondoped $(20 \%)$ carbon rods of $10 \mathrm{~mm}$ in diameter.

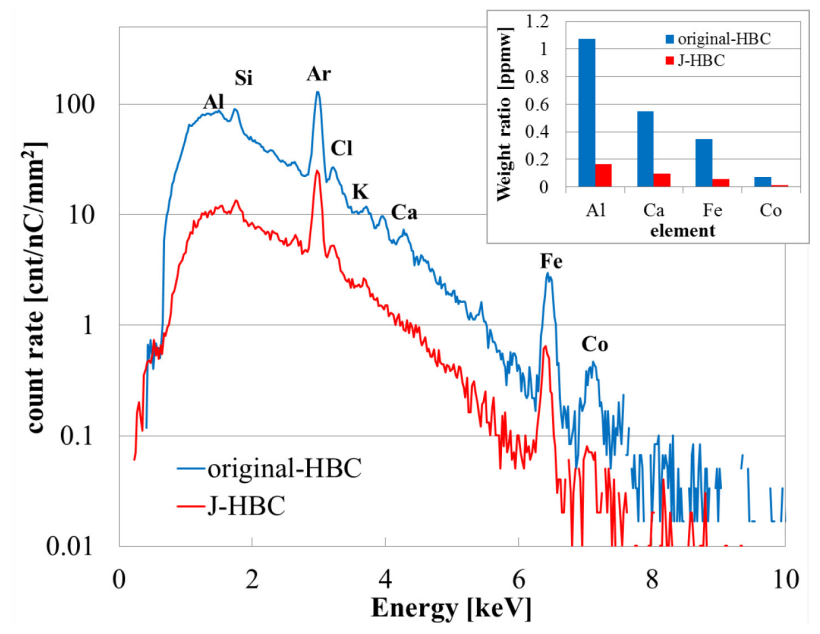

Fig. 6. Comparison of RBS spectra of original HBC foil and J-HBC foil (lower-left), and concentrations of trace impurities (upper-right).

\subsubsection{Identification of trace impurities by $\mu P I X E$}

Impurities in the foil, especially high- $Z$ material, cause large-angle beam scattering and generate radionuclides in the foil. Thus, efforts to reduce impurities in the foil are very important from the viewpoint of using the foil in a high-power proton beam accelerator, such as JPARC. To identify and evaluate quantitatively the trace impurities, the HBC foils were analysed with the $\mu$ PIXE method. The lower-left graph in figure 6 shows a comparison of the $\mu \mathrm{PIXE}$ spectra of the original-HBC foil and the J-HBC foil. The same elements were identified in both foils. Argon, which exhibited relatively high peak signals, was identified as background noise by the measurement system. Other elements were estimated quantitatively as shown in the upper-right bar chart of figure 6. The concentrations of trace impurities in the J-HBC foil are considerably lower than those in the original-HBC foil, but the underlying reason is not clear. Both foils were fabricated according to almost the same manufacturing methods and using the same deposition apparatus except for the vacuum device treatment. These trace impurities are considered to mainly contaminate from the boron-doped electrode [11]. As a consequence, we will investigate the quantitative evaluation of the trace impurities depending on the arc-discharge condition.

\subsubsection{Endurance test with ion beam irradiation}

To investigate the endurance against beam irradiation, we performed an argon ion beam irradiation test by using the TIARA $400-\mathrm{kV}$ ion implanter. In this test, the foil was irradiated with a $300-\mathrm{keV} \mathrm{Ar}^{+}$beam. It was found that the foil thickness of $330 \mu \mathrm{g} / \mathrm{cm}^{2}$ was too thick for the $\mathrm{Ar}^{+}$beam to pass through. Then, a considerably thinner foil with a thickness of $20 \mu \mathrm{g} / \mathrm{cm}^{2}$ was adopted to adjust an energy deposition in the foil to suit that at the J-PARC RCS. The thin J-HBC foil was mounted on an aluminium plate with a hole of $10 \mathrm{~mm}$ in diameter. After the J-HBC foil was irradiated for $30 \mathrm{~min}$ with a beam of $4 \mathrm{~mm}$ in diameter and beam current of $50 \mathrm{nA}$, its surface structure changed, but the foil did not break. Figure 7 shows the foil surface after beam irradiation, as observed with a digital microscope (KEYENCE VHX-6000). The irradiation spot was flattened and primary crack pattern on the surface disappeared. By contrast, the surrounding area showed radial wrinkles but the primary crack pattern is still present on the surface. This transition of the surface structure was the same as that observed in case of the original HBC foils [14]. It was analytically shown that radial wrinkles in a thin elastic sheet arise from compressive stresses in a region between inner and outer annular boundaries [17]. The radial wrinkles on the foil around the irradiation spot are ascribable to the same principle. Specifically, beam irradiation should cause an increase in density to the foil. And it brings a volumetric shrinkage and imposes inward radial force along the beam boundary.

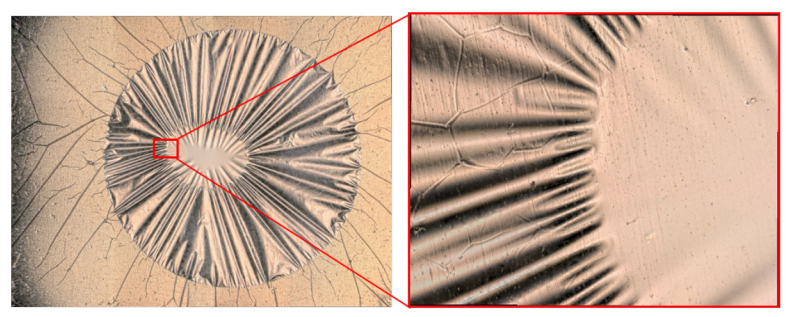

Fig. 7. Surface structure after $\mathrm{Ar}^{+}$beam irradiation, as observed using digital microscope.

\subsection{Demonstration of J-HBC foil for RCS user operation}

The J-HBC foils were verified to have good performance equivalent to that of the original $\mathrm{HBC}$ foil by means of offline tests in the TIARA. Thus, the J-HBC foils were installed in the RCS for user operation in September 2017. The RCS delivered protons to MLF users with a beam power of $300 \mathrm{~kW}$ and a repetition rate of $25 \mathrm{~Hz}$, and to NU users with equivalent beam power of $700 \mathrm{~kW}$ and four shots at intervals of $2.48 \mathrm{~s}$. Before use of the J-HBC foil for user operation, short duration trial tests were carried out under each beam condition. Figure 8 shows a photograph of the transition of the J-HBC foil during beam irradiation in the J-PARC RCS. Photo (a) 
shows the initial state of the foil. After the trial test with the MLF user beam for 30 minutes, the irradiation spot hollowed out slightly, as in photo (b). After the trial test with the NU user beam for $5 \mathrm{~min}$, the foil deformed clearly, as in photo (c). However, its performance of the stripper foil remained unaffected. Then, the J-HBC foil was successfully used in the user operation for 1 week, as in photo (d). This great result is a big milestone for HBC foil manufacture in J-PARC. In the future, we will attempt to use the J-HBC foil for long-term operation starting October 2018 to evaluate its performance.
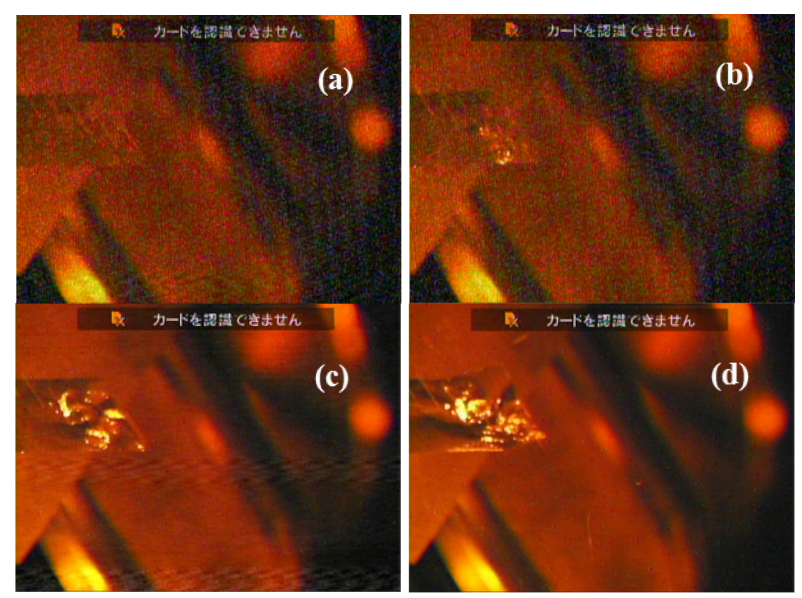

Fig. 8. Observation of J-HBC foil during user beam operation. (a) before irradiation, (b) trial test with MLF user beam for 30 min, (c) trial test with NU user beam for 5 min, and (d) after MLF and NU user operation for 1 week.

\section{Summary}

J-PARC RCS has been using the HBC stripper foils since the start of its operation, and the good performance of the stripper foil has been verified. Beam power for user beam operation has been increasing smoothly, and the HBC foil can be used for long-term user operation. The deposition apparatus for the HBC foil was relocated from the KEK Tsukuba site to the JAEA Tokai site in 2017 and we started fabrication of a new HBC foil at the JAEA Tokai site (J-HBC). Before using the J-HBC foil for user beam operation, offline beam test for the performance evaluation in the TIARA took place. The obtained test results are as follows: The cluster structure of the J-HBC foil is the same as that of the original HBC foil. The boron-to-carbon ratio of the J-HBC foil is slightly lower than that of the original HBC foil. The concentrations of trace impurities in the J-HBC foil are very low compared to those in the original $\mathrm{HBC}$. Moreover, the endurance of the J-HBC foil against beam irradiation is the same as that of the original $\mathrm{HBC}$ foil. After the performance verification of the J-HBC foil, it was employed successfully in RCS user operation for 1 week. This great result is a major milestone for HBC foil manufacture at J-PARC. In the future, we will attempt to use the J-HBC foil for long-term operation to evaluate its performance. As a consequence, we have not only been carrying out stable supply for the J-PARC user operation but also done research and development on HBC foil to improve the performance and quality.
The authors would like to thank O. Takeda, R. Saeki, and M. Mutoh for technical assistance with foil manufacturing. The foil evaluation and analysis were mainly carried out in QST TIARA. We are grateful to Dr. T. Sato and M. Koka for special support for experiment and analysis of $\mu$ PIXE, Dr. T. Taguchi for the analysis of the TEM and useful discussions, and Dr. S. Yamamoto for the analysis of the RBS and excellent support in TIARA. We are also grateful to Prof. Y. Irie for valuable advice from his rich experience and knowledge on accelerators including stripper foils. Finally, we would like to express our special thanks to Dr. I. Sugai for his guidance and encouragement. He pioneered the HBC stripper foil development and the success of this research is down to him.

\section{References}

1. High-intensity Proton Accelerator Project Team: JAERI Report No. JAERI-Tech 2003-044

2. High-intensity Proton Accelerator Project Team: KEK Report No. 2002-13.

3. M. Yoshimoto, P.K. Saha, Y. Yamazaki, M. Kawase, R. Saeki, N, Hayashi, K. Yamamoto, H. Hotchi, T. Ishiyama, M. Kinsho, I. Sugai, and Y. Irie, J. Phys.: Conf. Ser. 417, 012073 (2013).

4. I. Sugai, Y. Takeda, M. Oyaizu, H. Kawakami, Y. Irie, Y. Arakida, K. Hara, H. Hattori, K. Kawasaki, J. Kamiya, M. Kinsho, Nucl. Instrum. Methods Phys. Res., Sect. A 561,16-23 (2006).

5. I. Sugai, M. Oyaizu, and Y. Takeda, JP2007545272A, 2005-11-15. (in Japanese)

6. I. Sugai, M. Oyaizu, and Y. Takeda, WO2007/058224 A1, 2007-5-24. (in Japanese), (https://patents.google.com/patent/WO2007058224 A1/en, accessed 2020-01-10, (in English))

7. A. Tatami, M. Tachibana, M. Murakami, T. Yagi, M. Murakami, AIP.: Conf. Proc. 1962, 030005 (2018).

8. H. Hotchi, M. Kinsho, K. Hasegawa, N. Hayashi, Y. Hikichi, S. Hiroki, J. Kamiya, K. Kanazawa, M. Kawase, F. Noda, $\uparrow$ M. Nomura, N. Ogiwara, R. Saeki, P. K. Saha, A. Schnase, Y. Shobuda, T. Shimada, K. Suganuma, H. Suzuki, H. Takahashi, T. Takayanagi, O. Takeda, F. Tamura, N. Tani, T. Togashi, T. Ueno, M. Watanabe, Y. Watanabe, K. Yamamoto, M. Yamamoto, Y. Yamazaki, H. Yoshikawa, and M. Yoshimoto, Phys. Rev. ST Accel. Beams 12, 040402 (2009).

9. K. Hasegawa, H. Oguri, T. Ito, E. Chishiro, K. Hirano, T. Morishita, S. Shinozaki, H. Ao, K. Ohkoshi, Y. Kondo, J. Tamura, S. Yamazaki, T. Hori, F. Sato, Y. Nemoto, I. Koizumi, N. Ouchi, N. Kikuzawa, A. Ueno, A. Miura, S. Fukuta, A. Yoshii, K. Sato, A. Ozone, Y. Sawabe, Y. Kawane, H. Ikeda, Y. Ito, Y. Kato, K. Kikuchi, F. Hiroki, T. Takayasu, T. Usami, M. Yanai, K. Tadokoro, K. Ohsawa, F. Naito, Y. Liu, Z. Fang, T. Sugimura, K. Futatsukawa, K. Ikegami, M. Kawamura, K. Nanmo, Y. Fukui, T. Miyao, T. Maruta, A. Takagi, JPS Conf. Proc. 8, 011012 (2015). 
10. H. Hotchi, H. Harada, N. Hayashi, S. Kato, M. Kinsho, K. Okabe, P. K. Saha, Y. Shobuda, F. Tamura, N. Tani, Y. Watanabe, K. Yamamoto, M. Yamamoto, and M. Yoshimoto, Phys. Rev. ST Accel. Beams 20, 060402 (2017).

11. Y. Yamazaki, M. Yoshimoto, P.Saha, M. Kinsho, T. Taguchi, S. Yamamoto, and I. Sugai, JRNC, 3, 305, 859-864 (2015).

12. I. Sugai, M. Oyaizu, Y. Takeda, H. Kawakami, H. Hattori, and K. Kawasaki, Nucl. Instrum. Methods Phys. Res., Sect. A 590, 32-36 (2008).

13. I. Sugai, et. al, Nucl. Instrum. Methods Phys. Res., Sect. A 613, 457-461 (2010).

14. Y. Yamazaki, M Yoshimoto, O Takeda, M Kinsho, T Taguchi, S Yamamoto, T Kurihara, and I Sugai, J. Phys.: Conf. Ser. 417, 012071 (2013).

15. I. Sugai, T. Hattori, H. Suzuki, H. Kinishita, H. Kato, K. Yamazaki, Nucl. Instrum. Methods Phys. Res., Sect. A 265, 376 (1988).

16. M. Naterm SIMNRA User's Guide, Max PlanckInstitute-fur-Plasmaphysik, ver.7.01 (2017).

17. B. Davidovitch, R. D. Schroll, D. Vella, M. AddaBedia, and E. Cerda, Proc. Natl. Acad. Sci. U.S.A. 108, 18227 (2011). 